\title{
Etude de la ventilation de la vallée de la Sarine en Gruyère
}

\begin{abstract}
Summary
A case study has been carried out in the Sarine valley in Gruyère (Switzerland) in order to improve the understanding of thermotopographic winds over a complex terrain. Apart from field observations (fixed network, soundings, tracking of constant level balloons), two models have been tested with the aim to apply these models in areas with a similar topography. The physical or laboratory model was able to create realistic mountain or valley winds. The hydrostatic numerical model (MESOCONV) has been used to determine threedimensional wind patterns for two important anticyclonic weather situations (with NE or SW synoptic winds).
\end{abstract}

\section{Introduction}

De nombreuses études sur le transport et la diffusion de polluants sont actuellement menées dans le monde. Elles nécessitent notamment une bonne connaissance de certains paramètres météorologiques (vent, stabilité thermique etc.).

Or, les situations anticycloniques sont préoccupantes au niveau de la qualité de l'air, car elles se caractérisent dans nos contrées par une mauvaise ventilation. Ces situations favorisent le développement de brises. Dans une région accidentée, il existe plusieurs types de courants thermiques qui se superposent, ce qui rend leur étude plus difficile.

L'étude menée en Gruyère a eu pour cadre une grande vallée préalpine (Sarine) à topographie relativement complexe.' Ce projet a eu pour but:

- de mieux comprendre les particularités des écoulements dans une topographie accidentée et de confirmer les observations faites par d'autres auteurs à ce sujet.

- d'expérimenter des modélisations tridimensionnelles des écoulements en vue d'obtenir un modèle performant pour cette région accidentée; ce modèle doit pouvoir être transposé dans d'autres régions semblables.
Pour cela, cette étude a comporté de nombreuses mesures sur le terrain qui ont servi de base aux modélisations des écoulements expérimentées. Elle a déjà fait l'objet de plusieurs publications (FALLOT et al., 1988 et 1989). Dans ce papier, nous présenterons la méthodologie adoptée et quelques résultats marquants.

\section{Le cadre de l'étude}

La Gruyère constitue un tronçon de la vallée de la Sarine situé au pied des Alpes à environ $20 \mathrm{~km}$ au sud de Fribourg (Suisse). Elle se subdivise en deux régions morphologiquement distinctes (voir fig. 4):

- En Haute-Gruyère, la vallée de la Sarine est relativement encaissée et entourée de sommets dont l'altitude dépasse $2000 \mathrm{~m} / \mathrm{mer}$; le fond de la vallée est à environ $750 \mathrm{~m} / \mathrm{mer}$.

- En Basse-Gruyère, la vallée s'élargit et forme un bassin dont le fond est occupé par un lac d'accumulation ( 680 $\mathrm{m} / \mathrm{mer}$ ). Les reliefs environnants sont nettement plus doux qu'en Haute-Gruyère: leur altitude atteint 1200 $\mathrm{m} / \mathrm{mer}$ à l'ouest et $1700 \mathrm{~m} / \mathrm{mer}$ à l'est. Deux passages relient ce bassin au Plateau Suisse: l'un au nord du lac (Rossens) et l'autre à l'ouest de Bulle (Vaulruz). Enfin, une importante vallée latérale (Jogne) débouche en Gruyère à l'est de Bulle.

\section{Les mesures sur le terrain}

Deux types de mesures ont été réalisées:

- Un réseau fixe de mesures a enregistré en continu les vents et les températures de l'air en quelques endroits représentatifs durant 2 ans. Il a permis de suivre l'évolution des courants au gré des situations météorologiques rencontrées.

- Des campagnes de mesures intensives ont complété les données du réseau fixe dans l'espace pour certaines journées favorables au développement de brises. Ces campagnes ont notamment comporté:

Jean-Michel Fallot, géographe, Institut de Géographie, Pérolles, $\mathrm{CH}-1700$ Fribourg

\footnotetext{
' Cette recherche a été financée par le Fonds national suisse de la recherche scientifique (subside No 2.838-0.86). Plusieurs collaborateurs ont participé à cette étude: $A$. M. de BUMAN, P.A. BÄRISWYL et Prof. M. ROTEN de I'IGUF, Dr. M. BENISTON et Dr. J.A. HERTIG de I'EPFL.
}

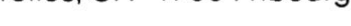


* des mesures complémentaires des vents près du sol en vue d'obtenir des cartes de ventilation pour l'ensemble de la Gruyère;

* des sondages verticaux afin de prospecter la dimension verticale et de voir l'interaction entre les différents courants thermiques et les vents synoptiques;

* des lancers de ballons stabilisés en vue d'obtenir des trajectoires typiques des courants et de mettre en évidence les multiples actions de la topographie sur les écoulements.

L'ensemble de ces mesures nous ont notamment permis de dresser des cartes schématiques typiques des écoulements pour les brises de vallée et de montagne (fig. 1). Ces cartes vont servir de référence à la présentation des résultats ci-après.

\subsection{Les écoulements près du sol}

La figure 2 représente l'évolution journalière de l'intensité moyenne des brises mesurée à $10 \mathrm{~m} / \mathrm{sol}$, ceci pour chaque saison et à 3 endroits représentatifs. Les stations de Grandvillard et d'Echarlens sont typiques de la Gruyère et des vallées alpines en général: les brises de vallée assu- rent une meilleure ventilation que celles de montagne, notamment au printemps et en été. En saison froide, l'accumulation d'air froid sur le Plateau Suisse et le faible réchauffement des reliefs enneigés entravent le développement des brises de vallée.

Les brises de montagne ne soufflent que faiblement et irrégulièrement en toute saison. Le bassin de la BasseGruyère favorise une accumulation d'air froid et un freinage des écoulements près du sol; celui-ci est ressenti jusqu'en Haute-Gruyère. Les mesures complémentaires des vents étendues à l'ensemble de la région ont d'ailleurs montré que de nombreuses poches d'air froid immobile se forment çà et là en Gruyère. Seuls, les endroits situés aux débouchès de la Basse-Gruyère (Rossens et Vaulruz) se caractérisent par des brises de montagne très sensibles: l'air froid accumulé en Gruyère s'écoule en direction du Plateau Suisse. En conséquence, les variations d'intensité des courants entre le jour et la nuit s'atténuent à ces endroits-là.

Les cartes schématiques des écoulements (fig. 1) montrent que la ventilation de la Gruyère par situation anticyclonique est dominée par les flux régionaux remontant et redescendant la vallée de la Sarine. Les vents de pente et les courants des vallées latérales n'ont qu'une incidence
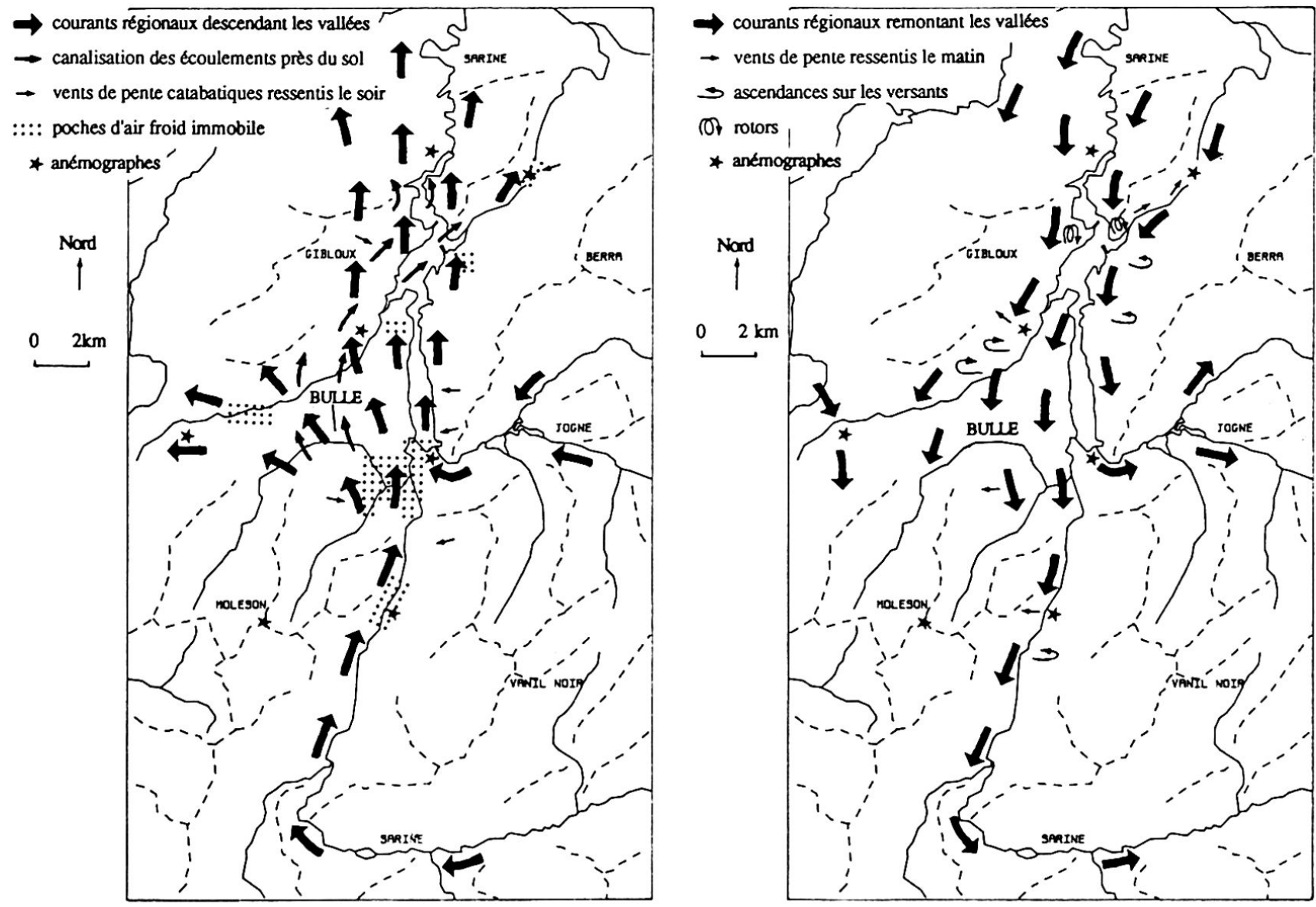

Figure 1 Cartes schématiques des écoulements nocturnes (à gauche) et diurnes (à droite) mesurés sur le terrain par situation anticyclonique estivale. 


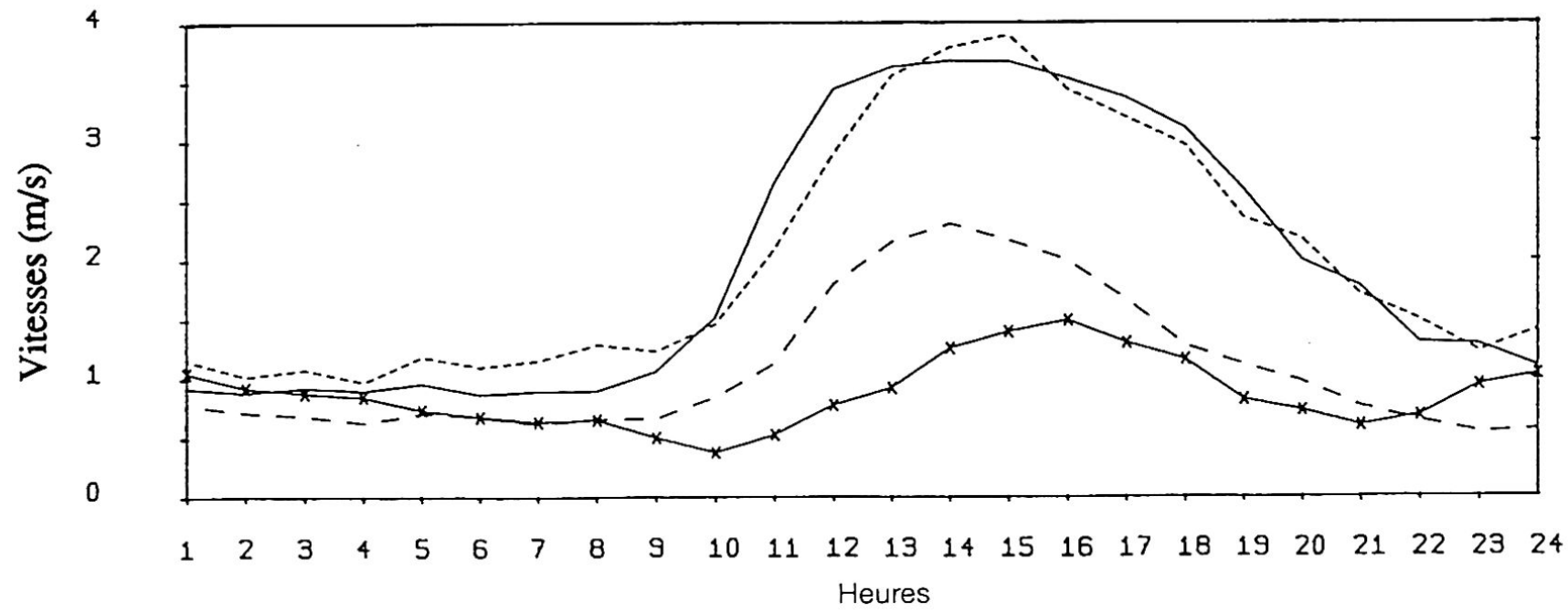

ECHARLENS

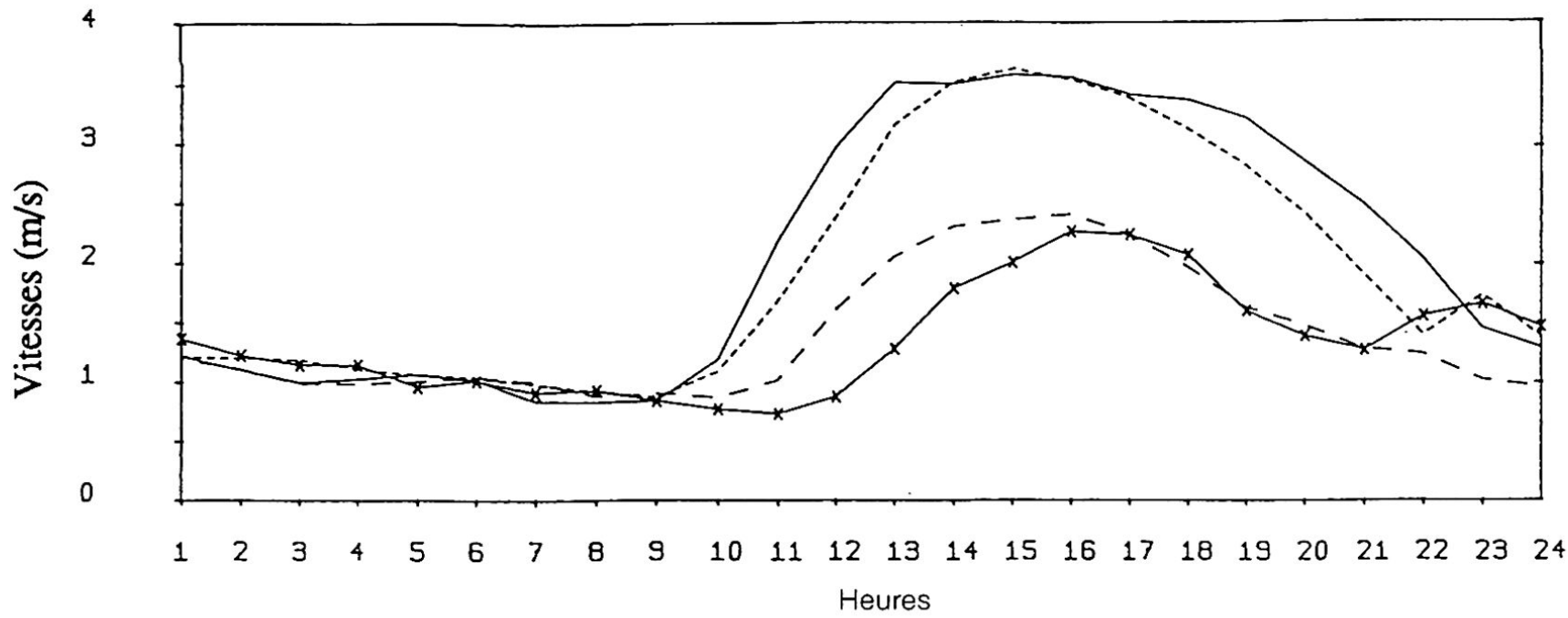

ROSSENS

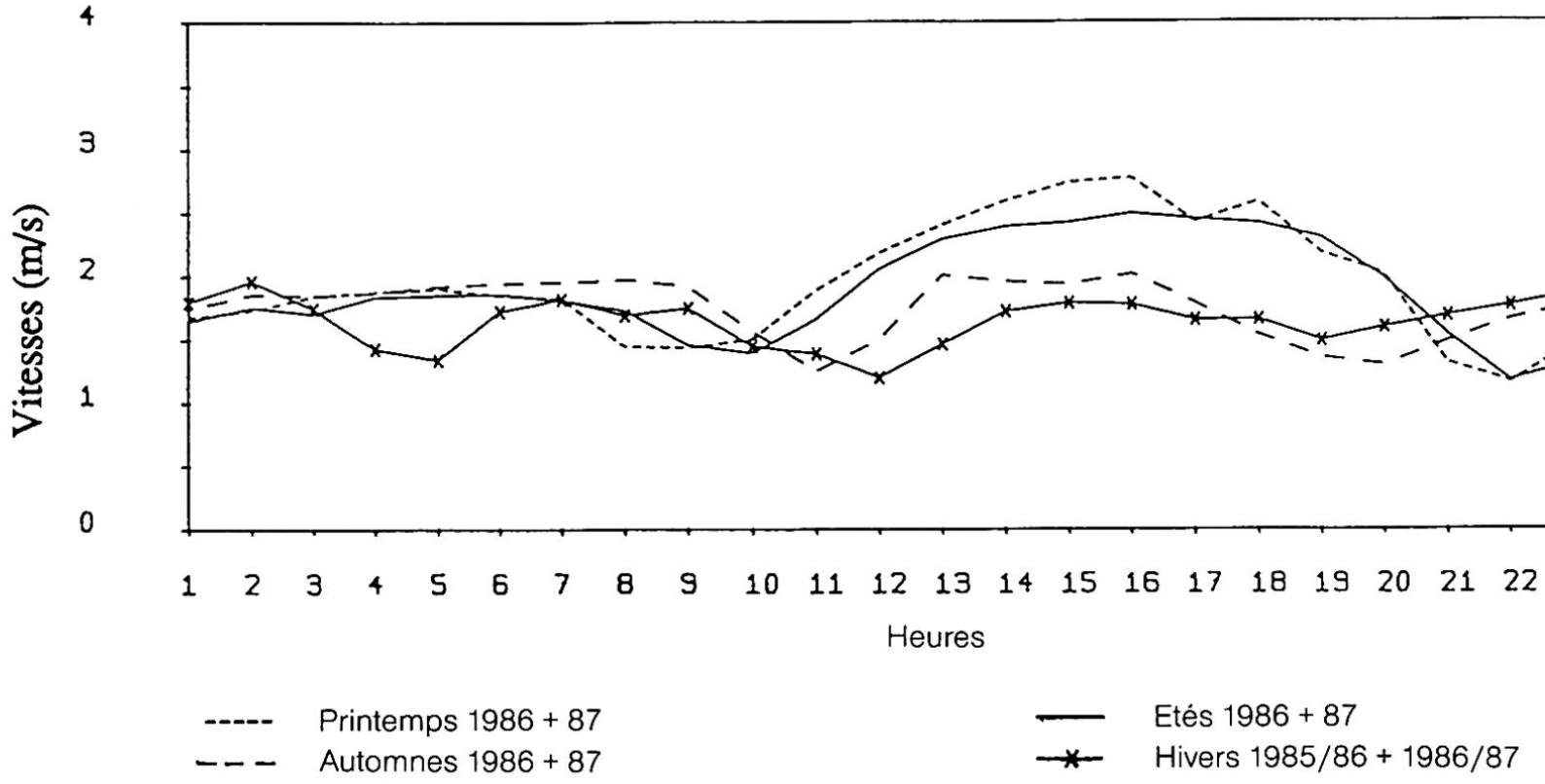

Figure 2 Vitesses moyennes des brises mesurées à trois endroits représentatifs de la Gruyère: Grandvillard (HauteGruyère), Echarlens (Basse-Gruyère) et Rossens (débouché septentrional de la Basse-Gruyère). 
limitée sur la direction des flux régionaux. La canalisation des écoulements par la topographie locale est plus efficace.

Nous disposons également d'une station de mesure sur un sommet préalpin (Moléson: $2000 \mathrm{~m} / \mathrm{mer}$ ). Contrairement à ce qui se passe au fond de la vallée, les courants soufflent le plus fortement durant la nuit et le plus faiblement en cours de matinée. Ceci rejoint les observations faites ailleurs pour lesquelles une explication a déjà été donnée (STURMAN, 1987).

Le soir, la renverse des courants est très rapide en un endroit donné. Elle s'opère d'abord en Haute-Gruyère et les brises de montagne s'étendent ensuite progressivement vers l'aval: il faut compter en moyenne 2,5 heures pour une distance de $20 \mathrm{~km}$.

Par contre, la renverse du matin est partout plus hésitante: les courants ne tournent que progressivement et cette période est propice à l'apparition de vents de pente locaux. Ceux-ci peuvent souffler jusqu'à 2 ou 3 heures en certains endroits avant d'être effacés par la brise de vallée.

Durant les 2 années de mesures, en 1986 et 1987, un régime de brises est apparu entre 30 et $40 \%$ des cas selon les endroits en Basse-Gruyère; ce pourcentage atteint même $50 \%$ des cas en Haute-Gruyère.

Toutes les situations anticycloniques ne se caractérisent pas systématiquement par un régime de brises en Gruyère. Ces dernières peuvent être perturbées par des paramètres extérieurs tels que la direction et la force des vents synoptiques, la nébulosité ou le gradient de pression régional existant le long du Plateau Suisse. Des analyses statistiques plus poussées ont montré que la persistance des brises en Gruyère dépend en premier de la provenance des vents synoptiques liés au champ de pression général régnant sur l'Europe.

\subsection{Les vents dans la troisième dimension}

Les sondages verticaux ont permis de caractériser la couche de brises dans la troisième dimension en plusieurs endroits et pour différentes journées (fig. 3). Deux grands types de situations anticycloniques doivent être distinguées, à savoir:

- celles caractérisées par un afflux d'air continental à basse altitude du nord à est,

- et celles caratérisées par un afflux d'air subtropical du sud à ouest.

Les profils thermiques et l'épaisseur des brises diurnes et nocturnes ne diffèrent guère d'un endroit à l'autre de la Gruyère. Par contre, la direction et la force des brises varient plus ou moins fortement en fonction des particularités de la topographie.

Les profils de températures potentielles mettent en évidence deux couches d'air, tant le jour que la nuit:

- On repère à basse altitude, une couche d'air influencée par les variations diurnes des températures du sol; une couche convective se développe le jour sur une épaisseur de plusieurs centaines de mètres et elle est remplacée la nuit par une inversion thermique.
- Plus haut, les températures n'évoluent que peu entre le jour et la nuit; c'est l'atmosphère libre caractérisée par un gradient thermique légèrement stable.

Les profils des vents laissent apparaitre trois couches d'air, tant le jour que la nuit:

- Les brises de vallée et de montagne soufflent dans les 500 à 700 premiers mètres de l'atmosphère. Leur épaisseur semble conditionnée par l'altitude des cols qui découpent les reliefs entourant le bassin préalpin de la haute Sarine.

- Elles sont généralement surmontées de vents faibles et variables jusqu'à l'altitude des sommets préalpins (environ $2000 \mathrm{~m} / \mathrm{mer}$ ). Nous n'avons pas trouvé trace de courants compensatoires ("antiwinds») dans cette couche tels que ceux décrits dans la littérature (BUTTNER \& THYER, 1966).

- Plus haut soufflent les vents synoptiques dont la direction et la force évoluent en fonction de la situation météorologique générale; lorsqu'ils se renforcent, une zone d'accélération des courants apparait juste au-dessus des reliefs préalpins.

Les profils des températures et des brises varient sensiblement d'un jour à l'autre en fonction de la provenance des vents synoptiques et des masses d'air. Ainsi, un afflux d'air continental du nord-est à basse altitude a pour effet: - d'atténuer le développement de la couche convective diurne et de renforcer la brise de vallée soufflant dans le même sens;

- de s'opposer à l'écoulement de la brise de montagne et d'atténuer l'ampleur de l'inversion thermique: l'air froid est redistribué sur une plus grande épaisseur.

Par contre, un afflux d'air subtropical du sud-ouest a un effet inverse:

- Il affaiblit quelque peu la brise de vallée et favorise un développement plus important de la couche convective: en raison de l'instabilité croissante de l'air, les profils des vitesses deviennent très turbulents.

- Il favorise le développement d'une inversion thermique très prononcée dans les basses couches et renforce la brise de montagne soufflant dans le même sens.

Des sondages effectués toutes les heures à l'aide d'un ballon captif ont montré que le soir, la renverse des courants a lieu pratiquement simultanément sur une épaisseur de 500 à 700 mètres (= couche des brises régionales). $\mathrm{Ceci}$ contraste avec la croissance progressive de la couche d'inversion durant la nuit. L'épaisseur de la brise de montagne reste constante et seule son intensité continue d'augmenter durant la nuit.

D'autres sondages verticaux effectués le long des pentes ont permis de déceler le soir des brises de pente catabatiques. Elles disparaissent durant la nuit, étant probablement effacées par l'inversion régionale de la vallée et par les brises de montagne plus puissantes. Le jour, nous avons découvert plusieurs déviations locales des courants qu'il faut plutôt attribuer à des particularités locales de la topographie (effet dynamique) qu'à une influence des vents de pente (effet thermique). Il semble que, en ac- 

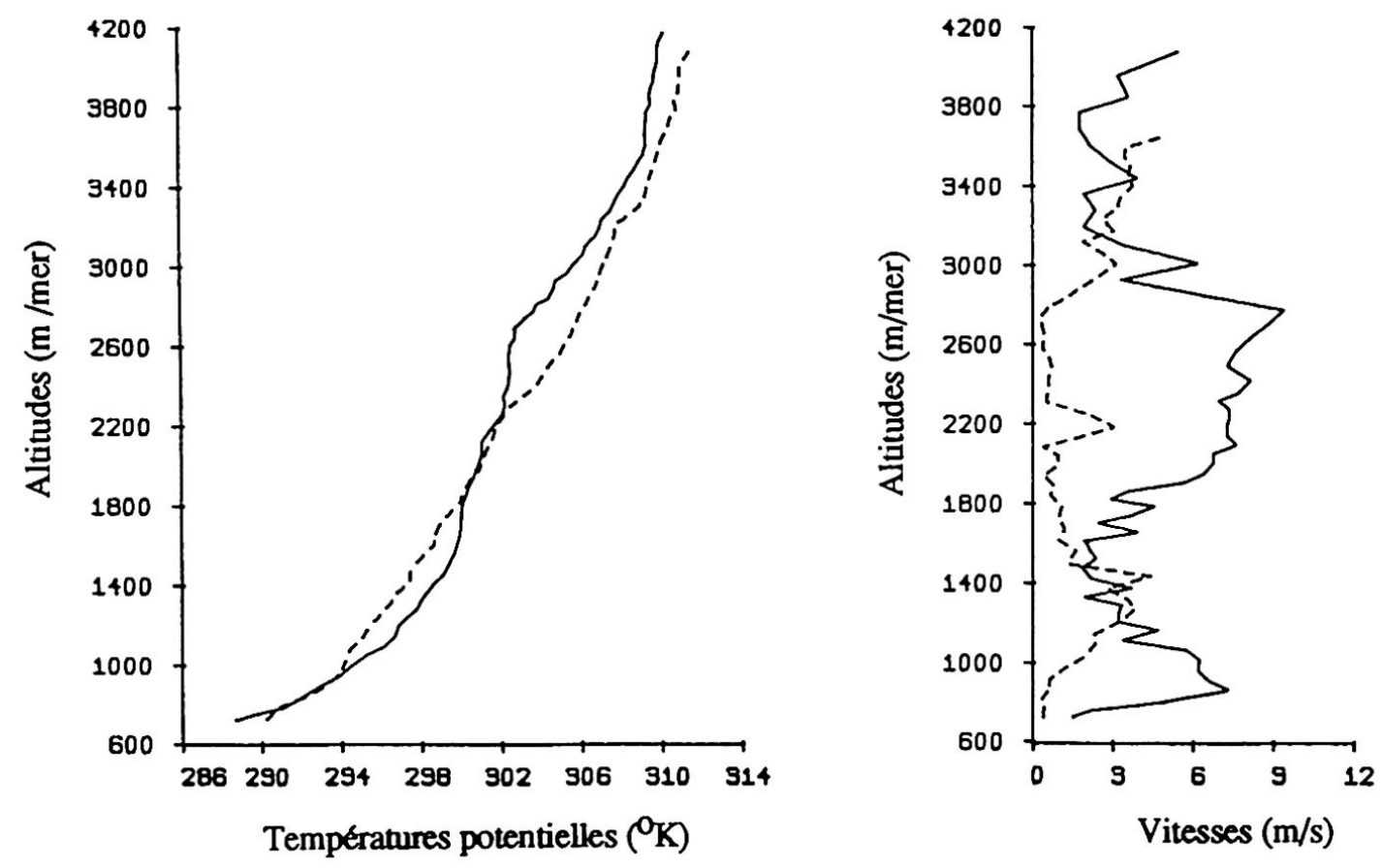

$\downarrow$ Vent du nord

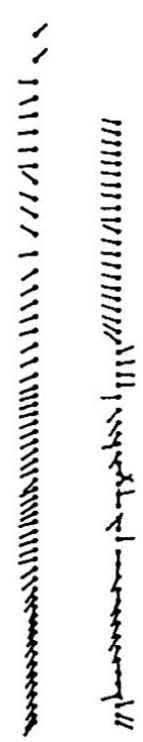

12

Figure 3a Profils des températures potentielles (à gauche) et des vents (à droite) mesurés en Gruyère (Granvillard) à l'aube de deux situations anticycloniques estivales typiques.
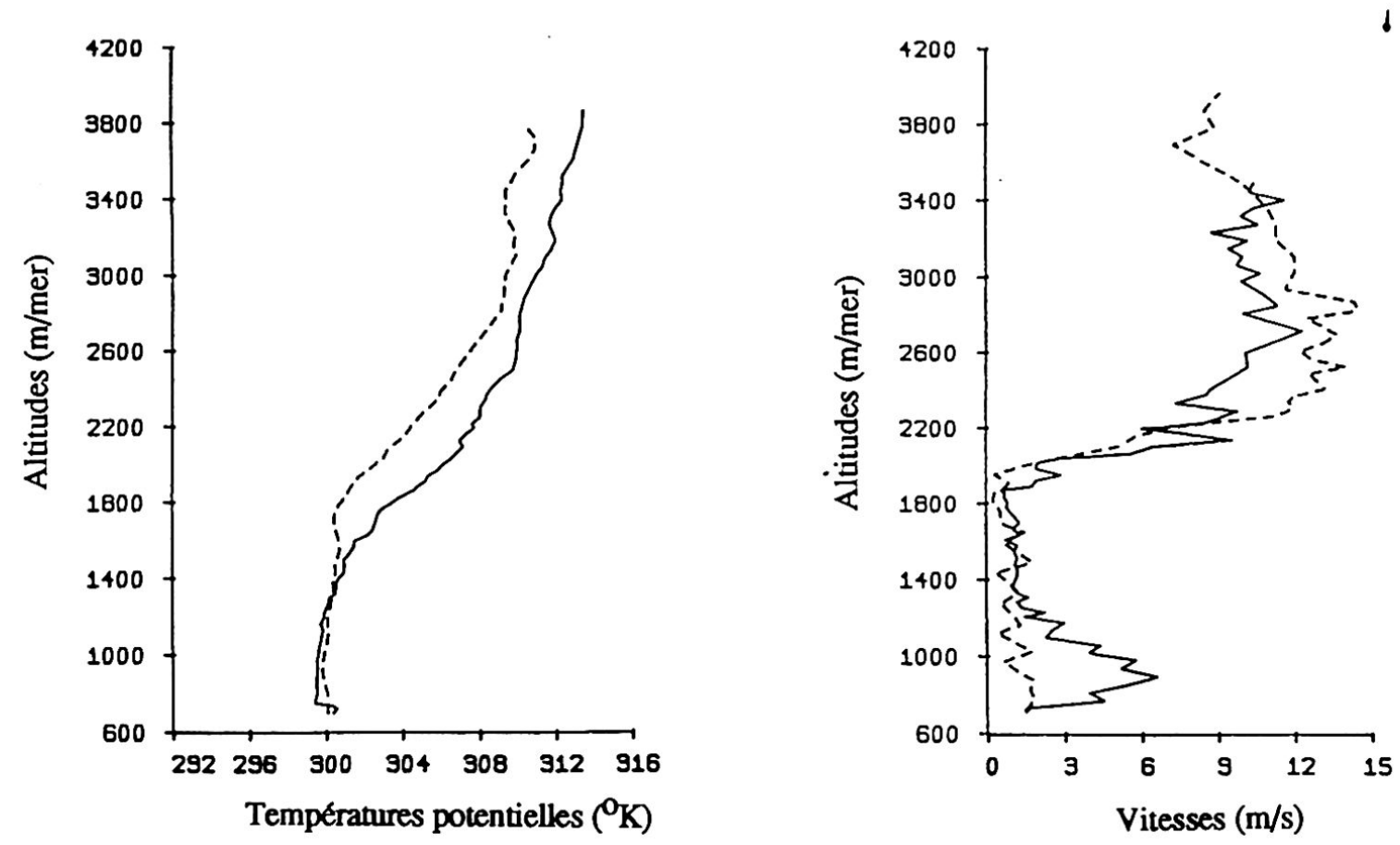

Vent du nord

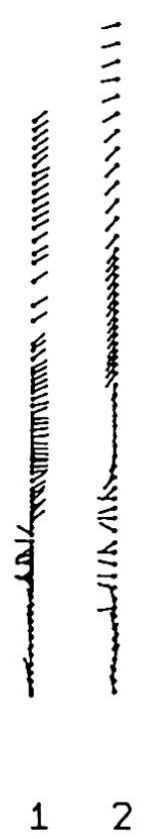

$-\quad$ Situation anticyclonique du NE: $29.8 .198515 \mathrm{~h}(1)$
-

Figure 3b Profils des températures potentielles (à gauche) et des vents (à droite) mesurés en Gruyère (Broc) l'après-midi de deux situations anticycloniques estivales typiques. 
cord avec les trajectoires de ballons stabilisés, l'action thermique des versants se limite, l'après-midi, à des ascendances emportées par la brise de vallée.

Les trajectoires de ballons stabilisés ont mis en évidence de nombreux mouvements verticaux d'origine thermique et dynamique se développant dans l'air instable des brises de vallée: rotors hélicoïdaux le long des versants, subsidences (rotors) sous le vent des arêtes, etc. En revanche, aucun mouvement vertical significatif n'affecte l'air stable des brises de montagne.

\section{Modélisations tridimensionnelles des écoulements}

Afin de compléter les données récoltées sur le terrain et de déterminer les écoulements dans les trois dimensions pour l'ensemble de la région étudiée, nous avons effectué des modélisations physique et numérique des écoulements. Les deux types de modélisation ont prouvé leur performance pour d'autres études. Il nous a paru néanmoins utile de les expérimenter dans cette région topographiquement complexe. En outre, les brises de vallée n'ont été que rarement simulées jusqu'à présent. Si la physique des écoulements est bien connue et prise en compte par les modèles, il importe encore de résoudre certains paramètres géographiques. Pour notre étude, les interactions entre les vallées et l'influence des régions environnantes non incluses dans les modèles (= conditions aux limites) nécessitent une attention particulière; une mauvaise estimation de ces paramètres a une incidence non négligeable sur les courants régionaux.

\subsection{Modélisation physique des écoulements}

Elle consiste en une reproduction des écoulements sur des maquettes topographiques à petite échelle disposées dans une soufflerie aérodynamique ou dans une enceinte climatologique. Cette technique a été rendue possible par la mise au point de lois dites de similitude autorisant le passage de la nature au modèle physique réduit. Elles ont été décrites par HERTIG (1986). Les essais de modélisation physique ont eu lieu dans les installations du LASEN-EPFL. Elles n'auraient pu être réalisées sans la collaboration étroite du Dr. J. A. HERTIG et de M.P. LISKA qui ont mis au point cette technique de simulation en Suisse. Pour la simulation des brises de vallée et de montagne, il convient de reproduire des écoulements stratifiés en altitude, de pouvoir chauffer et refroidir la surface de la maquette et de travailler en température potentielle.

La maquette topographique de la Gruyère a été construite en feuille d'aluminium à l'échelle $1: 5000$. Elle a été installée dans une enceinte climatologique circulaire qui permet la reproduction d'écoulements stratifiés en altitude. Le refroidissement de la surface du modèle est assuré par de l'azote liquide injecté sous le modèle. Des lampes infra-rouges accrochées au plafond permettent de réchauffer la surface du modèle.

Plusieurs paramètres météorologiques sont mesurés à l'aide de capteurs. Nous pouvons notamment effectuer des profils verticaux des températures et des vents sur le modèle, qui peuvent ensuite être comparés avec ceux obtenus sur le terrain. Enfin, l'injection de fumées sur la maquette permet de visualiser les courants dans les trois dimensions: des cartes d'écoulement schématiques ont pu être dressées (fig. 4).

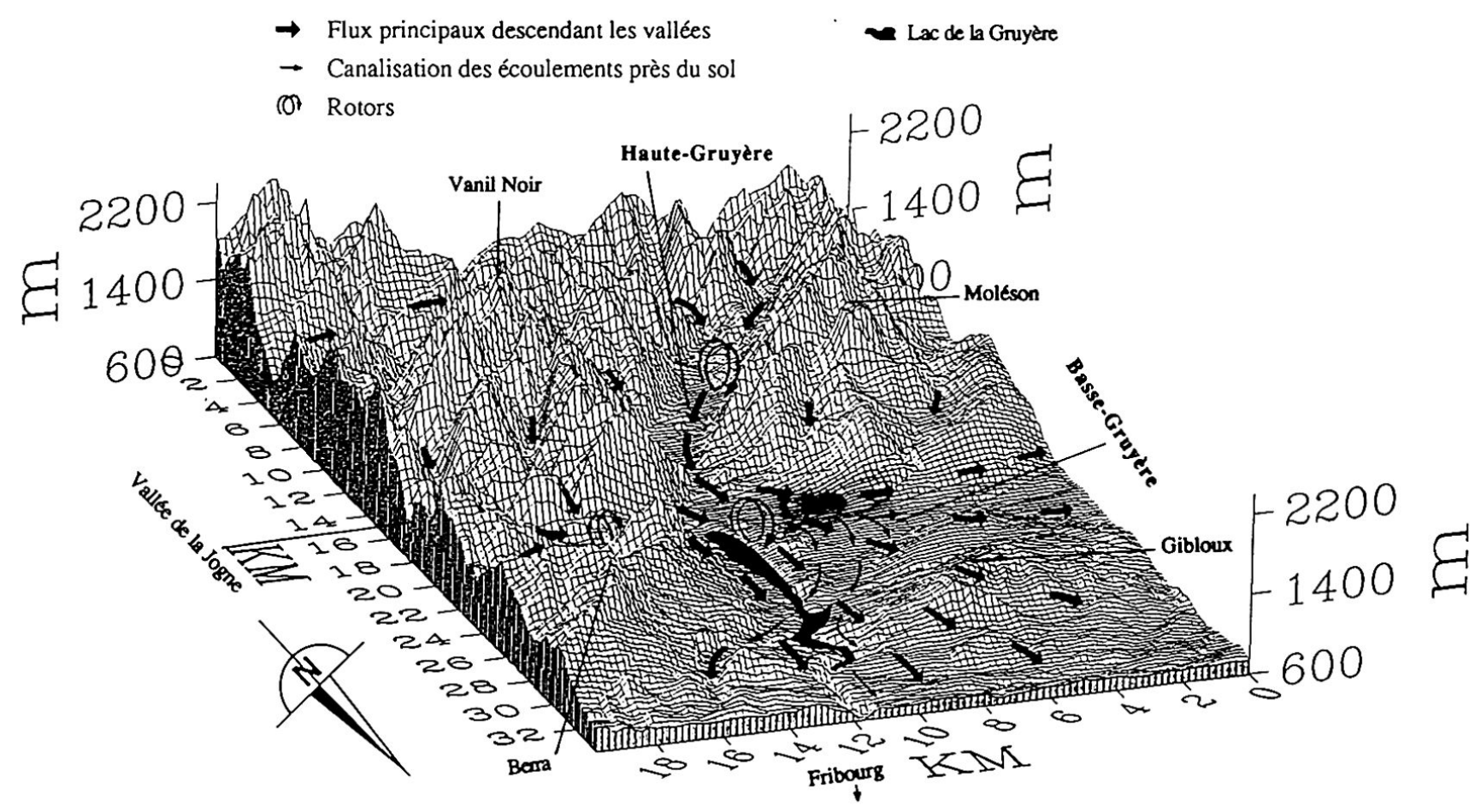

Figure 4 Carte schématique des écoulements nocturnes simulés sur le modèle physique de la Gruyère. 


\subsubsection{Simulation des brises de montagne}

Elles naissent au contact d'un sol plus froid que l'air environnant et correspondent à un écoulement de gravité. A l'échelle régionale, les vallées drainent l'air froid descendant des montagnes vers les plaines situées en aval. Il suffit donc pour reproduire les brises nocturnes de refroidir la surface du modèle tout en respectant les lois de similitude.

Mais il faut encore tenir compte du freinage des écoulements nocturnes en Gruyère que provoque durant la nuit le remplissage d'air froid du Plateau Suisse situé an aval. Nous avons réussi à inclure cette influence et à caler le modèle pour certaines journées anticycloniques bien documentées sur le terrain.

Sur les cartes schématiques des écoulements obtenues pour la brise de montagne (fig. 4), on retrouve l'essentiel des observations faites sur le terrain. Mais l'échelle retenue pour le modèle ne permet pas de reproduire les poches d'air froid locales pelliculaires. De même, nous ne trouvons pas trace de brises de pente catabatiques. Par contre, nous avons découvert sur le modèle l'existence de rotors à axe horizontal aux endroits où les flux de deux vallées d'une certaine importance confluent; ces rotors ne se forment que pour des brises puissantes.

Nous avons également effectué des simulations plus théoriques en vue de mieux comprendre certains mécanismes météorologiques et de voir le rôle de certains paramètres sur les profils des brises. Nous avons notamment fait varier l'ampleur du refroidissement nocturne pour un vent en altitude du nord, de direction opposée à la brise de montagne. Comme attendu, l'intensité de la brise varie plus ou moins linéairement avec l'ampleur du refroidissement nocturne (fig. 5). Pour une inversion thermique bien développée, les profils des vitesses sur le modèle ressemblent à ceux des brises de pente catabatiques approximés par PRANDTL (1942): les vitesses maximales se rencontrent dans la moitié inférieure de la couche, comme dans la nature (cf. fig. 3). Lorsque le refroidissement nocturne s'atténue, les profils des brises présentent une forme plus irrégulière.

L'épaisseur de la brise augmente rapidement avec le refroidissement nocturne, puis se stabilise lorsque celui-ci atteint une certaine valeur. Si le refroidissement nocturne se poursuit, seule l'intensité de la brise continue à croître de manière sensible: ceci confirme l'évolution des courants mesurée durant la nuit sur le terrain.

\subsubsection{Simulation des brises de vallée}

Elles naissent le jour en présence d'un sol chaud que l'air environnant et correspondent à un écoulement plutôt turbulent. A l'échelle régionale, les reliefs surchauffés provoquent un mouvement d'air généralisé et les courants remontent les vallées. La simulation des brises de vallée est plus complexe que celles de montagne, car il ne suffit pas simplement de réchauffer la surface du modèle pour les générer. Il faut encore:

- stratifier l'air en altitude de manière à créer un couvercle thermique et à canaliser les courants ascendants le long des pentes et des vallées;
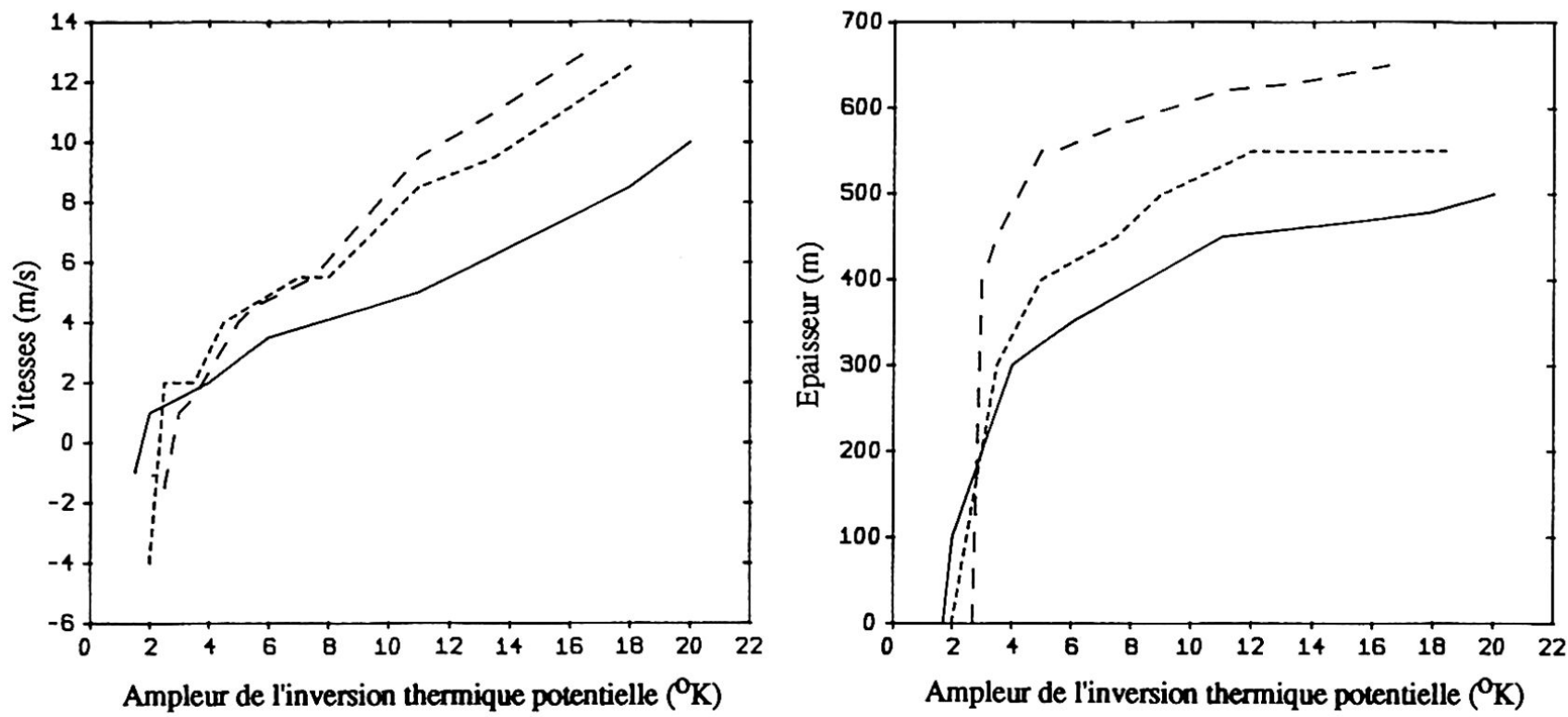

\footnotetext{
_--- vent synoptique du Nord: vitesse de $9 \mathrm{~m} / \mathrm{s}$, écoulement neutre - - - vent synoptique du Nord: vitesse de $3 \mathrm{~m} / \mathrm{s}$, écoulement stratifié
}

Figure 5 Modèle physique:évolution des vitesses maximales (à gauche) et de l'épaisseur (à droite) des brises de montagne en Gruyère en fonction de l'ampleur du refroidissement nocturne. 
- assurer l'évacuation de l'air chaud en ménageant des ouvertures dans les parois latérales de l'enceinte, au sommet des vallées, afin de générer un appel d'air généralisé de l'aval; sinon, les écoulements sont bloqués dans l'enceinte.

Le réglage des conditions aux limites s'avère plus délicat, car il s'agit de contrôler aussi bien les débits des flux d'aval entrant en Basse-Gruyère que les débits sortant à l'extrémité des vallées préalpines. Malgré ces difficultés, nous avons réussi à caler le modèle pour les situations anticycloniques du nord-est et du sud-ouest. Les profils et les cartes schématiques des écoulements diurnes obtenus sur le modèle s'apparentent aux mesures faites sur le terrain.

Les simulations effectuées ont confirmé que la stratification thermique de l'air en altitude influence grandement les profils des brises de vallée. Ceux-ci sont le mieux développés et s'apparentent aux profils des brises de montagne lorsque de l'air stable en altitude limite la croissance de la couche convective. L'intensité des brises de vallée croît avec l'ampleur du réchauffement du sol. Mais lorsque celui-ci atteint une certaine ampleur, l'augmentation de l'instabilité thermique et de la turbulence dans les basse couches commencent à détruire et à affaiblir les brises de vallée: les profils deviennent plus irréguliers et les vitesses maximales ne se rencontrent plus systématiquement dans la moitié inférieure de la couche des brises (cf. fig. 3).

Nous avons également pu simuler sur le modèle la renverse du matin pour laquelle nous disposions de peu de mesures sur le terrain. Avec le réchauffement du modèle, les brises de montagne s'affaiblissent et la renverse des courants ne s'effectue que progressivement. Des vents de pente ascendants se développent sur les versants exposés et des ascendances apparaissent alors au-dessus des reliefs les plus bas; le niveau atteint par ces ascendances dépend de la stabilité thermique de l'air recouvrant les vallées.

En Haute-Gruyère, les vents de pente ascendants sont bloqués au niveau des sommets et ramenés au centre de la vallée; ils contribuent ainsi directement au réchauffement diurne de son atmosphère. En revanche, l'élargissement de la vallée en Basse-Gruyère et l'altitude plus modeste des reliefs environnants ont pour effet que les ascendances d'air chaud au-dessus de ces reliefs sont emportées par le vent en altitude. Elles ne participent donc pas comme en Haute-Gruyère à la dissipation (par le haut) de l'inversion thermique et au réchauffement diurne de l'atmosphère de la vallée; celui-ci s'effectue uniquement à partir du sol, par convection, comme dans une région plane. Cette différence dans le réchauffement de l'air d'une vallée et d'une plaine a déjà été observée précédemment (WHITEMANN, 1982; BREHM \& FREYTAG, 1982).

Si nous poursuivons le réchauffement du modèle, les brises de vallée s'établissent d'abord dans les vallées préalpines, puis sur l'ensemble de la Gruyère. Lorsque ces brises se renforcent et que l'instabilité thermique croît dans les basses couches, les vents de pente disparaissent peu à peu; ils font place à des ascendances verticales d'air chaud ("pompes thermiques») au-dessus des versants.

\subsection{Modélisation numérique des écoulements}

Un modèle météorologique tridimensionnel à mésoéchelle MESOCONV a été développé par M. BENISTON pour simuler les écoulements et la formation de nuages convectifs au-dessus de la mer. Ce modèle a ensuite été adapté à la problématique des brises en milieu accidenté; il est décrit notamment dans BENISTON (1987).

Le modèle MESOCONV résout les équations primitives (approximations de Boussinesq) pour les composantes horizontales du vent, la température potentielle (équation thermodynamique), l'humidité spécifique et l'eau liquide sous forme de goutelettes de nuages et de gouttes de pluie. Pour l'étude de la Gruyère, le modèle en différences finies contient 32000 points de grilles répartis en $32 \times 50 \times 20$ points dans les trois directions $x$, y et $z$; la distance entre chaque point de grille est de $500 \mathrm{~m}$ horizontalement et de $100 \mathrm{~m}$ verticalement. Ce modèle est hydrostatique et son sommet se situe à près de $2000 \mathrm{~m} / \mathrm{sol}$ (environ $2600 \mathrm{~m} / \mathrm{mer}$ ); seules des situations avec peu d'activité en plan vertical peuvent être simulées efficacement. Les mesures faites sur le terrain fournissent les données initiales au modèle; elles permettent aussi de contrôler l'exactitude des simulations effectuées. Les conditions aux limites latérales du modèle doivent être soigneusement règlées pour reproduire correctement les entrées et les sorties d'air aux bons endroits. Ceci se fait en imposant des termes de pression aux limites latérales qui dirigeront le courant dans le sens désiré. Pour cela, une bonne connaissance des courants sur le terrain s'avère nécessaire.

M. BENISTON a réussi à simuler avec succès les brises de montagne et de vallée pour les situations anticycloniques du nord-est et du sud-ouest. Le modèle MESOCONV complète les résultats du modèle physique en permettant de mieux quantifier les paramètres météorologiques dans les trois dimensions. On peut notamment obtenir:

- des cartes précises des écoulements en plan à différents niveaux,

- des profils verticaux des températures et des vents à n'importe quel endroit,

- des coupes verticales des écoulements,

- des trajectoires de particules.

La figure 6 présente à titre d'exemple les cartes d'écoulement en plan obtenues à $250 \mathrm{~m} / \mathrm{sol}$ pour les brises de vallée et de montagne; ces cartes sont nettement plus détaillées que celles du modèle physique. Le modèle numérique MESOCONV permet aussi de calculer d'autres paramètres difficilement mesurables sur le terrain (composante w du vent, bilan de masse, etc.) Enfin, il peut être couplé à d'autres modèles développés pour le transport et la diffusion des polluants en milieu accidenté (BENISTON et al., 1989).

Par contre, le modèle physique prend mieux en compte la topographie. Malgré certaines approximations, les simulations sur les deux modèles ont permis de confirmer plu- 
t

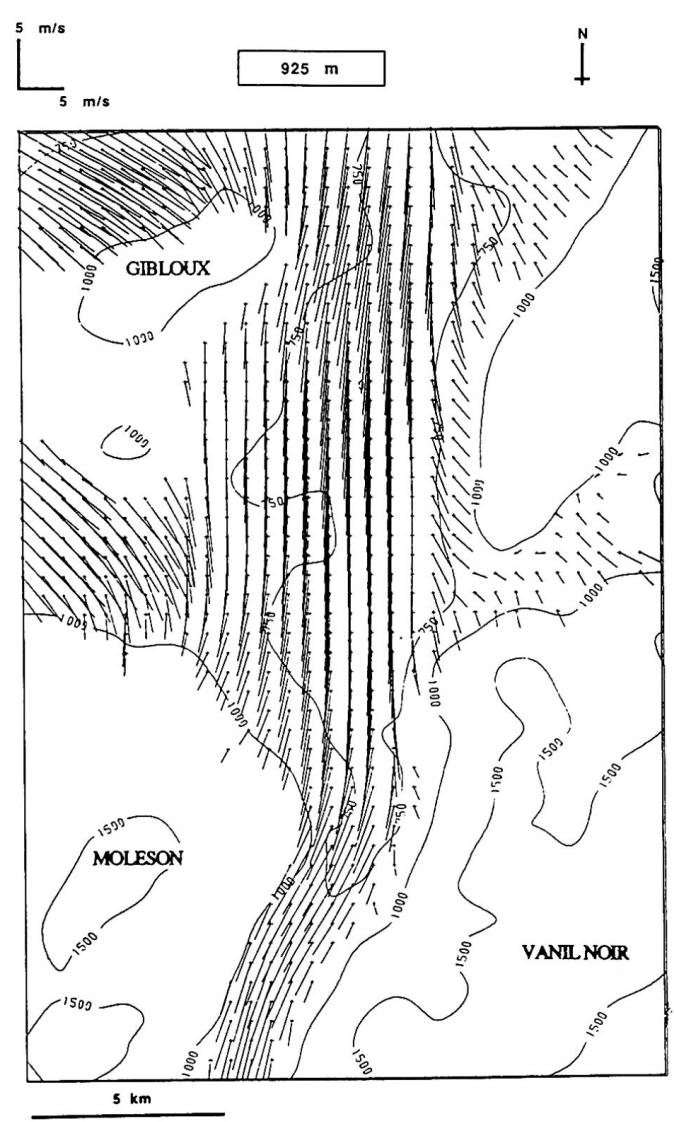

$\bigsqcup_{5 \mathrm{~m} / \mathrm{s}}^{5 \mathrm{~m} / \mathrm{s}} \stackrel{\mathrm{N}}{\mathrm{H}}$

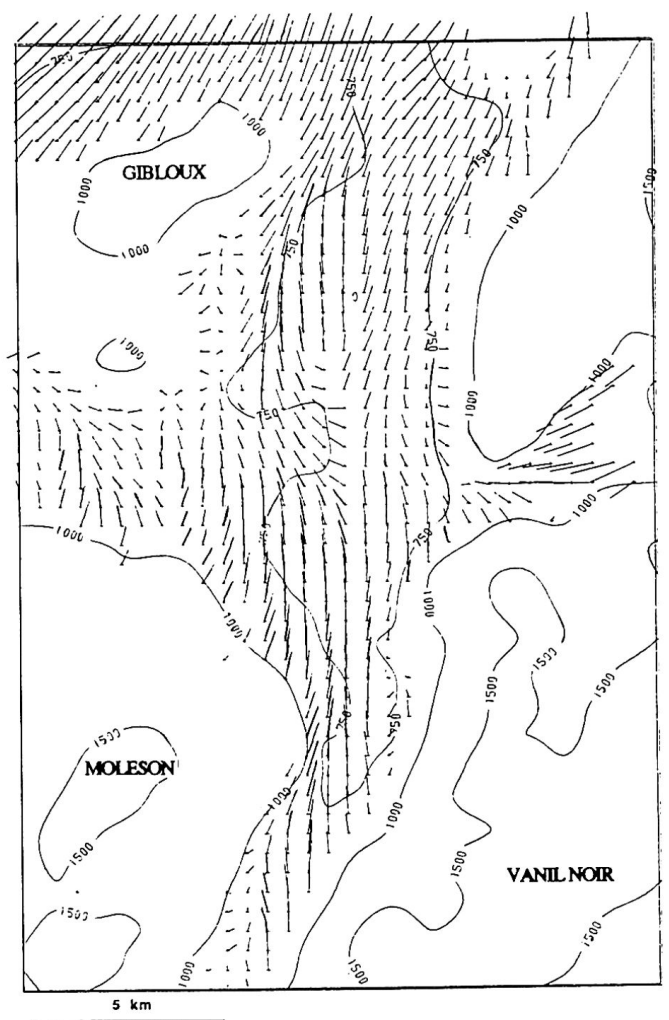

Figure 6 Modèle numérique MESOCONV: carte schématique des écoulements nocturnes (à gauche) et diurnes (à droite) en plan au niveau $925 \mathrm{~m} / \mathrm{mer}$ (= environ $250 \mathrm{~m} / \mathrm{sol}$ ). 
sieurs observations faites sur le terrain et de les compléter dans les trois dimensions. Ils ont aussi contribué à mieux comprendre certaines particularités des écoulements observées en milieu accidenté.

\section{Bibliographie}

BENISTON, M. (1987): A numerical study of atmospheric pollution over complex terrain in Switzerland. Energy Transformations and Interactions with Atmospheric Processes, M. Beniston \& R.A. Pielke (Ed.), D. Reidel Publ., 75-96.

BENISTON, M., D. RUFFIEUX and J.A. HERTIG (1989): Combined numerical and wind-tunnel studies of meteorology and air pollution episodes in a rural valley of Switzerland. Boundary Layer Meteorology, 48, 129-156.

BREHM, M. and C. FREYTAG (1982): Erosion on the nighttime thermal circulation in an alpine valley. Arch. Met. Geophys. Biokl. B31, 331-352.

BÜTTNER, K.J.K. and H.THYER (1966): Valley winds in Mt. Rainer area. Arch. Met. Geophys. Biokl. Ser. B, Bd 14 H-2, 125-147.

FALLOT, J. M., I. BÄRISWYL, A. M. de BUMAN, M. BENISTON et M. ROTEN (1988): Etude des brises thermiques d'une grande vallée préalpine suisse: la vallée de la Sarine en
Gruyère. Mesures sur le terrain et essais de modélisation des écoulements. Actes des journées de climatologie, Mont-Rigi, Belgique, 5-7 novembre 1987. Presses universitaires de Liège, 75-96.

FALLOT, J.M., P.A. BÄRISWYL, A.M. de BUMAN, M. BENISTON, et M. ROTEN (1989): Etude des brises nocturnes et diurnes d'une grande vallée préalpine en vue de l'établissement de modèles empiriques de ventilation applicables à d'autres vallées préalpines. Rapport final destiné au Fonds national de la recherche scientifique. Projet No. 2.838-0.86, 186 p. + annexes.

HERTIG, J. A. (1986): Modélisation des impacts de perturbations atmosphériques dans les basses couches de l'atmosphère. Rapport IENER-EPFL N511.134, Lausanne.

PRANDTL, L. (1942): Führer durch die Strömungslehre. F. Vieweg \& Sohn, Braunschweig.

STURMAN, A.P. (1987): Thermal Influences on airflow in moutainous terrain. Progress in Physical Geography, Vol. 11 (2), 182-206.

VERGEINER, J. and E. DREISETL (1987): Valley winds and slope winds, observations and elementary thoughts. Meteorol. Atmos. Phys. 36, 264-286.

WHITEMANN, C.D. (1982): Breakup of temperature inversions in deep mountain valleys. Part 1: Observations. J. Appl. Met, 21, p. 270-289. 